\title{
LEEP in the Family Practice Setting
}

\author{
David J. Lyman, MD, MPH, and Brent Morris
}

Background: We wanted to review our 7-year experience using the loop electrical excision procedure (LEEP) for the treatment of cervical dysplasia in a family practice residency setting in the rural South.

Methods: We conducted a retrospective study with data gathered from chart review of a mostly Medicaid and uninsured patient population of rural Southern women referred from outlying health departments or private practices within an 80 -mile radius. The women received follow-up Papanicolaou smears, and outcome measurements were either recurrence of dysplasia or at least 1 year with two negative Papanicolaou smears. Any surgical tissue obtained after LEEP was used to ascertain residual or recurrent dysplasia.

Results: Rates of disease recurrence and incomplete excision of cervical intraepithelial neoplasia grade 2/3 (CIN 2/3) compared favorably with results published by expert US gynecologists but were worse than those reported by European authors, who excise all CIN (CIN 1, CIN 2, and CIN 3).

Conclusion: CIN 2 and CIN 3 can be diagnosed and treated appropriately with LEEP in the setting of a family practice residency. (J Am Board Fam Pract 2003;16:204-8.)

Many family physicians trained in colposcopy also use the loop electrical excision procedure (LEEP) to treat cervical dysplasia or cervical intraepithelial neoplasia (CIN). This procedure can obviate the need for referral to a gynecologist for cold knife conization, the former reference standard for treating severe dysplasia. Callaway and Frisch ${ }^{1}$ are the most recent family physician colposcopists to confirm low referral rates (9\%) while managing 283 patients in their Cervical Dysplasia Clinic. The assumption, however, is that both the family physician and gynecologist achieve similar rates of positive tissue margins and disease recurrence after LEEP.

A review of the literature found a single multisite study published in 1995 by family physicians Ferris and colleagues. ${ }^{2}$ The primary inclusion criterion for this study was cervical dysplasia of any severity. One hundred thirty-two of 198 patients had at least one Papanicolaou smear and a repeated colposcopy after LEEP. Of the 132 patients, 119 (90.2\%) had no residual lesions on a single follow-up examination, although the authors did not distinguish between those patients with mild disease and those with moderate to severe dysplasia. Positive margins

Submitted, revised 15 May 2002.

From the Department of Family Medicine (DJL), and the School of Medicine (BM), University of Tennessee, Jackson. Address reprint requests to David J. Lyman, MD, MPH, Family Practice Residency Program, UT/Jackson Madison County General Hospital, 294 Summar Dr, Jackson, TN 38301 . (not defined) were found in $18.9 \%$ of LEEP specimens (17 of 90 ) and in $11.3 \%$ of LEEP-endocervical resection specimens ( 9 of 79 ). The authors reported a cure rate of $72.2 \%$ when excision of CIN was incomplete, a figure that compared favorably with results published in the medical literature. This pivotal study showed that family physicians well trained in colposcopy and LEEP could achieve rates of success comparable to those reported by other investigators.

Recommendations guiding the use of LEEP in cervical dysplasia are still evolving. First, positive margins in high-grade squamous cell dysplasia no longer demand immediate surgery. Although cold knife conization is always indicated when microinvasive squamous cell carcinoma or adenocarcinoma in situ is found in the specimen, patients with highgrade squamous cell dysplasia at the margins may be followed up using frequent cytologic testing or colposcopy to avoid repeated conizations or hysterectomy. ${ }^{3,4}$ In most patients ( $60 \%$ to $70 \%$ ), dysplasia will resolve despite positive margins. Second, a closer look at individual studies shows that some pathologists consider margins to be positive only if dysplasia involves the margin, whereas others use the term if either dysplasia or human papillomavirus infection is noted. With no consensus on the definition of positive margins, Jakus and colleagues $^{3}$ found that rates for margin positivity ranged from $8 \%$ to $85 \%$. Third, Dodson and Sharp ${ }^{4}$ have expressed concern that inexperienced physicians who learn this technique during a week- 
end seminar have inadequate training and insufficient patient volume to manage the complexities of CIN.

Finally, we wondered whether family practice residents and faculty doing colposcopy and LEEP could provide a level of care comparable to that of published investigators. We reviewed our 7-year experience (March 1993 to March 2000) in the Colposcopy Clinic at The University of Tennessee Family Medicine Center in Jackson. We hypothesized that family practice patients undergoing LEEP for treatment of CIN grade 2 (CIN 2) or CIN 3 in our clinic would achieve rates of cure comparable to that reported in published studies.

\section{Methods}

The University of Tennessee in Jackson is a wellestablished family medicine program during which residents are trained in colposcopy and the outpatient management of CIN. The colposcopy curriculum has been taught by family physician faculty and an obstetrician-gynecologist. Most of our rural west Tennessee patients are either uninsured or have Medicaid (TennCare) and are referred to us when cervical dysplasia is found during routine Papanicolaou smear screening. Our management of cytologic abnormalities mirrors current American College of Obstetricians and Gynecologists recommendations: any woman with a high-grade squamous intraepithelial lesion undergoes colposcopy, endocervical curettage, and appropriate cervical biopsies. ${ }^{5}$ If the entire lesion is seen and the limits of the transformation zone are noted, women with high-grade squamous intraepithelial lesions and all others with colposcopic or histologic evidence of CIN 2 or CIN 3 disease are considered candidates for LEEP-also termed an "electrical loop excision of the cervical transformation zone," or ELECTZ, by some authors.

Whenever possible, the transformation zone and all CIN lesions are excised to a depth of 7 to $8 \mathrm{~mm}$ by the single pass of a $2.0 \times 0.8$-cm wire loop electrode. If more than one pass is required to remove the transformation zone, the deepest portion of the excision is made over the central cervix in an attempt to preserve the squamocolumnar junction. All remaining negative areas (by Lugol stain) are excised with a 4 - to 5-mm margin of normal epithelium. If the endocervical specimen is positive for dysplasia or if white epithelium extends into the canal after the ectocervical excision, a second, deeper endocervical excision is obtained (a "cowboy-hat" or "top-hat" excision) by using the square $1.0 \times 1.0-\mathrm{cm}$ wire loop electrode. ${ }^{6}$ Finally, the ectocervical margins are cauterized with a 5-mm ball electrode to achieve hemostasis and destroy residual disease. ${ }^{6}$

Microinvasive or invasive squamous cell carcinoma and adenocarcinoma in situ are excluded from LEEP, because margin status is difficult to assess if the patient desires conservative treatment. ${ }^{4}$ Although 2\% to 3\% of LEEP specimens are unexpectedly found to harbor such lesions, none was found in our sample. After appropriate diagnosis and treatment, the patient is referred back to her primary care provider, usually the local health department or a resident family physician, with identical follow-up recommendations.

During the 7 -year period, 70 family practice patients received 73 LEEPs. The 3 patients who underwent immediate repeated wire loop excision for CIN 2 or CIN 3 at the surgical margins were included for analysis based on histologic and cytologic findings subsequent to the second procedure. A surgical margin was considered positive for dysplasia if the pathologist noted CIN or human papillomavirus infection at either the endocervical or ectocervical margin. A surgical margin was considered negative when so noted by the pathologist. When margin status was not noted, an attempt was made to clarify this oversight by contacting each signatory pathologist. No false-negatives were found by this tedious method, and we concluded that certain pathologists did not feel compelled to report negative margins in nonmalignant disease. Consequently, the few remaining reports with no mention of margin status were considered to be negative.

Patients were included for analysis if they had at least two negative Papanicolaou smears in a row or had evidence of recurrent or persistent dysplasia. Four patients with two negative Papanicolaou smears and negative margins were observed for less than 1 year but were included in the no-recurrence category for purpose of analysis. Recurrence was defined as none if the last two Papanicolaou smears were negative or if subsequent surgical tissue (ie, repeated LEEP, cold knife conization, or hysterectomy) had no evidence of residual CIN. A mild recurrence was considered to have occurred if $\mathrm{Pa}$ panicolaou smears showed atypical squamous cells 
Table 1. Recurrence of Dysplasia Relative to Margin

Result.

\begin{tabular}{lccc}
\hline Margin Result & $\begin{array}{c}\text { No } \\
\text { Recurrence } \\
\%(\text { No.) }\end{array}$ & $\begin{array}{c}\text { Mild } \\
\text { Recurrence } \\
\%(\text { No.) }\end{array}$ & $\begin{array}{c}\text { Severe } \\
\text { Recurrence } \\
\%(\text { No.) }\end{array}$ \\
\hline Positive margin & $75.0(18 / 24)$ & 0 & $25.0(6 / 24)$ \\
Negative margin & $84.8(39 / 46)$ & $15.2(7 / 46)$ & 0 \\
\hline
\end{tabular}

of undetermined significance (ASCUS) or a lowgrade squamous intraepithelial lesion or if histologically the tissue had evidence of residual CIN 1. Severe recurrence occurred if follow-up Papanicolaou smears showed a high-grade squamous intraepithelial lesion or if subsequent surgical specimens had any evidence of CIN 2 or greater.

\section{Results}

Our study cohort consisted of those 70 patients cared for exclusively at the Family Practice Center. These patients received their primary and follow-up care from residents or faculty physicians within the practice. The mean age of our patients was 29.0 years. Of the 24 patients $(34.3 \%)$ with positive tissue margins, endocervical margins were positive in 11 , ectocervical margins were positive in 3 , both margins were positive in 9, and positive margins were described but not localized in a single patient. Although endocervical glands showed CIN on occasion and might predict disease recurrence independent of margin status, our small sample size did not permit a separate analysis of this finding. Of the 24 patients with positive margins, 18 (75\%) had no recurrence of dysplasia, and $6(25 \%)$ had recurrent or persistent disease. All recurrences in these patients were rated as severe. Forty-six of the 70 patients $(65.7 \%)$ had negative surgical margins. Of these patients, $39(84.8 \%)$ had no recurrence, and 7 $(15.2 \%)$ had mild recurrences only. The rate of recurrence relative to margin status is summarized in Table 1.

In addition to excision of the transformation zone, 5 patients underwent a top-hat excision of the endocervical canal for indications outlined above. In four specimens, no residual dysplasia was found, LEEP surgical margins were also negative, and no recurrent dysplasia occurred. In the single positive top-hat procedure, endocervical margins of the LEEP specimen were also positive for dysplasia. This patient had no subsequent disease recurrence
Table 2. Contingency Table for No Recurrences.

\begin{tabular}{lccc}
\hline Margins & $\begin{array}{c}\text { Yes } \\
\text { Number }\end{array}$ & $\begin{array}{c}\text { No } \\
\text { Number }\end{array}$ & $\begin{array}{c}\text { Total } \\
\text { Number }\end{array}$ \\
\hline Negative & $39(37.46)^{*}$ & $7(8.54)$ & 46 \\
Positive & $18(19.54)$ & $6(4.46)$ & 24 \\
Total & 57 & 13 & 70 \\
\hline
\end{tabular}

*Numbers in parentheses are calculated.

Note: The observed $\chi^{2}=0.99$. If there were an effect at the $P=.25$ level, then the expected $\chi^{2}=1.32$; and at the $P=.05$ level, the expected $\chi^{2}=3.841$. Because the observed does not exceed the expected, the type of margin does not affect the frequency of no recurrence $(P>.25)$. Accept the null hypothesis that the frequencies are the same.

for a 2-year period. Excision of the endocervical canal did not appear to be of benefit in our small cohort.

Further data analysis was by chi-square contingency tables. In our study, the type of margin did appear to show some effect on the frequency of recurrence. but this difference did not reach the level of statistical significance (Table 2). Twentyfive percent of patients with positive margins (6 of 24) developed recurrent disease, whereas only $15.2 \%$ of patients with negative margins (7 of 46) developed recurrent disease $(0.25<P<.50)$. The type of margin did affect the severity of recurrence, however. Mild recurrence (as defined above) was found only in those patients who had negative surgical margins (Table 3). Severe recurrence (as defined above) occurred only in those patients who had residual CIN or changes secondary to human papillomavirus infection at the resected margin (Table 4).

\section{Discussion}

This review of our experience with LEEP in the family practice setting has obvious limitations.

Table 3. Contingency Table for Mild Recurrences.

\begin{tabular}{lccc}
\hline Margins & $\begin{array}{c}\text { Yes } \\
\text { Number }\end{array}$ & $\begin{array}{c}\text { No } \\
\text { Number }\end{array}$ & $\begin{array}{c}\text { Total } \\
\text { Number }\end{array}$ \\
\hline Negative & $7(4.6)^{*}$ & $39(41.4)$ & 46 \\
Positive & $0(2.4)$ & $24(21.6)$ & 24 \\
Total & 7 & 63 & 70 \\
\hline
\end{tabular}

*Numbers in parentheses are calculated.

Note: The observed $\chi^{2}=4.06$. If there were an effect at the $P=$ .05 level, then the expected $\chi^{2}=3.84$. Because the observed exceeds the expected, we conclude that the margin type affects the frequency of mild recurrence $(P=.05)$. Reject the null hypothesis that the frequencies are the same. 
Table 4. Contingency Table for Severe Recurrences.

\begin{tabular}{lccc}
\hline Margins & $\begin{array}{c}\text { Yes } \\
\text { Number }\end{array}$ & $\begin{array}{c}\text { No } \\
\text { Number }\end{array}$ & $\begin{array}{c}\text { Total } \\
\text { Number }\end{array}$ \\
\hline Negative & $0(3.94)^{*}$ & $46(41.4)$ & 46 \\
Positive & $6(2.06)$ & $18(21.94)$ & 24 \\
Total & 6 & 64 & 70 \\
\hline
\end{tabular}

*Numbers in parentheses are calculated.

Note: The observed $\chi^{2}=12.38$. If there were an effect at the $P=.005$ level, then the expected $\chi^{2}=7.88$. Because the observed exceeds the expected, we conclude the margin type affects the frequency of severe recurrence $(P=.005)$. Reject the null hypothesis that the frequencies are the same

First, excluded from analysis were an additional 109 patients treated by LEEP and referred back to their local health departments with identical follow-up recommendations. The intent of this article, however, was to study only those cases diagnosed, treated, and followed up within a family practice residency. Patients cared for by family physicians might be healthier or more compliant than those who receive episodic care through the health department. The true effect of selection bias can be addressed only through a more comprehensive study. Any conclusions drawn from this small study apply only to patients cared for in our practice. Second, the effect of different operators with varying levels of skill might be an unmeasured confounder. Treatment provided by a resident in training might be less efficacious than that provided by an experienced faulty operator.

Our $34.3 \%$ positive margin rate after LEEP is higher than that reported by Ferris and colleagues. ${ }^{2}$ In 1995 they reported a $15.4 \%$ rate of positive margins (26 of 169 LEEP specimens) when treating all grades of dysplasia. Are our results acceptable? In 1998 Hulman and colleagues ${ }^{7}$ published their experience with large-loop excision of the transformation zone (LLETZ). Although incomplete excision of CIN 1 occurred in only 6 of 109 patients (5.5\%) with low-grade histologic findings, the excision was incomplete in 106 of 143 patients (74\%) with CIN 3. During a 1.5 - to 3.5 -year follow-up period, CIN recurred in only $8.4 \%$ of patients who had CIN completely excised but recurred in $31.3 \%$ when CIN was incompletely excised. In 1999, Livasy and colleagues ${ }^{8}$ in North Carolina reviewed the LEEP slides of 200 women receiving treatment for CIN 3; they found high-grade changes at the margins in 106 (53\%) specimens, with endocervical gland involvement an independent risk for subse- quent abnormal cytologic findings. Conversely, in Greece Paraskevaidis and colleagues ${ }^{9}$ cared for 782 patients with all grades of CIN and found margin involvement in only $68(8.7 \%)$ of patients, and uncertain margin status in only 24 (3.1\%) patients. These studies suggest that those who treat all CIN with LEEP report lower rates of margin and endocervical gland involvement than those who perform LEEP for only high-grade disease.

Our failure to achieve statistical significance in Table 2 (15.2\% recurrent or persistent disease if margins were negative compared with $25 \%$ if margins were positive) in part reflects our small sample size. At least 2 patients, however, originally classified as having mild recurrences (ASCUS or lowgrade squamous intraepithelial lesions) were found to be free of infection when subsequent Papanicolaou smears obtained elsewhere were reviewed. The short interval between LEEP and the detection of mild recurrences occurred only in patients with negative margins, suggesting delayed resolution rather than failure of therapy. Also, various authors have described healing artifacts mistaken for dysplasia during the first 6 months after LEEP. ${ }^{9,10}$ Finally, infection with human papillomavirus is known to regress spontaneously in many women, although the time required for residual disease to clear is not known. ${ }^{11,12}$ Perhaps the high rates of cure reported by authors who perform LEEP as treatment for CIN 1 simply reflect the natural course of short-lived, low-grade human papillomavirus infection. It is not known whether aggressive surgical management of squamous intraepithelial lesions cures an infection with human papillomavirus. ${ }^{11}$ Consideration of subsequent cytologic abnormalities would therefore have been more appropriate before classifying our mild recurrences as LEEP failures.

Is cytologic testing alone adequate for follow-up of CIN 2 and CIN 3 treated with LEEP? Gardeil and colleagues ${ }^{13}$ in 1997 provided convincing evidence that completely excised CIN 3 can be safely followed up with cytologic testing alone. Subsequent authors have noted that with recurrent dysplasia abnormal cytologic findings are observed within the first year, regardless of margin status. ${ }^{8,9,14}$ Dietrich and colleagues ${ }^{14}$ most recently reviewed the results of 509 LEEPs at their institution. A study group of 298 patients was sampled cytologically at 3 and 6 months and underwent colposcopy at 6 months. Although high-grade 
changes on initial sampling were highly predictive of residual dysplasia, a single negative Papanicolaou smear at 3 months did not necessarily predict a cure. Of 174 patients with negative cytologic findings at 3 months after LEEP, an additional 14 patients $(8 \%)$ had dysplasia on repeated cytologic sampling at 6 months. Although all cases of dysplasia were detected cytologically within 2 to 12 months after LEEP, the authors concluded that, based on their retrospective review, patients with histologic risk factors for recurrence (margin or glandular involvement with dysplasia) should be examined colposcopically and cytologically at a 6-month follow-up visit. There are, however, no prospective randomized studies comparing cytologic testing with colposcopy after LEEP, an acknowledged weakness of current recommendations. ${ }^{14}$

Finally, unlike other sexually transmitted diseases, human papillomavirus infection does not receive active public health case management. Cervical cancer will kill 4,100 women in the United States in 2003, and rural areas in the South have the highest mortality rates. ${ }^{15,16}$ Recent investigators have found a high prevalence of oncogenic human papillomavirus DNA in all degrees of CIN, and a low prevalence in specimens with human papillomavirus infection changes only (koilocytosis). ${ }^{12}$ If this finding is reaffirmed, LEEP might become the treatment of choice for all degrees of cervical dysplasia. Our small study suggests that family physicians trained to treat CIN with LEEP can achieve rates of cure comparable to those reported by other investigators.

Statistical analysis was provided by Wayne Wofford, $\mathrm{PhD}$, of Union University, Jackson, Tenn.

\section{References}

1. Callaway P, Frisch L. Does a family physician who offers colposcopy and LEEP need to refer patients to a gynecologist? J Fam Pract 2000;49:534-6.

2. Ferris DG, Hainer BL, Pfenninger JL, Zuber TJ, DeWitt DE, Line RL. Electrosurgical loop excision of the cervical transformation zone: the experience of family physicians. J Fam Pract 1995;41:337-44.

3. Jakus S, Edmonds P, Dunton C, King SA. Margin status and excision of cervical intraepithelial neoplasia: a review. Obstet Gynecol Surv 2000;55:520-7.

4. Dodson MK, Sharp HT. Uses and abuses of the loop electrosurgical excision procedure (LEEP). Clin Obstet Gynecol 1999;42:916-21.

5. Kurman RJ. Cervical cytology: evaluation and management of abnormalities. Technical bulletin 183 . Washington, DC: American College of Obstetricians and Gynecologists, 1993:330-6.

6. Epperson WJ, Pfenninger JL. Electrosurgery for cervical intraepithelial neoplasia. The Female $\mathrm{Pa}$ tient 1998;23:33-49.

7. Hulman G, Pickles CJ, Gie CA, Dowling FM, Stocks PJ, Dixon R. Frequency of cervical intraepithelial neoplasia following large loop excision of the transformation zone. J Clin Pathol 1998;51:375-7.

8. Livasy CA, Maygarden SJ, Rajaratnam CT, Novotny DB. Predictors of recurrent dysplasia after a cervical loop electrocautery excision procedure for CIN-3: a study of margin, endocervical gland, and quadrant involvement. Mod Pathol 1999;12:233-8.

9. Paraskevaidis E, Lolis ED, Koliopoulos G, Alamanos Y, Fotiou S, Kitchener HC. Cervical intraepithelial neoplasia outcomes after large loop excision with clear margins. Obstet Gynecol 2000;95(6 Pt 1):82831.

10. Saha A, Maresh M. Women are often followed up too soon after treatment for cervical intraepithelial neoplasia. BMJ 1996;312:640.

11. Ho GY, Bierman R, Beardsley L, Chang CJ, Burk RD. Natural history of cervicovaginal papillomavirus infection in young women. N Engl J Med 1998;338: 423-8.

12. Adams E, Berkova Z, Daxnerova Z, Icenogle J, Reeves WC, Kaufman RH. Papillomavirus detection: demographic and behavioral characteristics influencing the identification of cervical disease. Am J Obstet Gynecol 2000;182:257-64.

13. Gardeil F, Barry-Walsh C, Prendiville W, Clinch J, Turner MJ. Persistent intraepithelial neoplasia after excision for cervical intraepithelial neoplasia grade III. Obstet Gynecol 1997;89:419-22.

14. Dietrich CS 3rd, Yancey MK, Miyazawa K, Williams DL, Farley J. Risk factors for early cytologic abnormalities after loop electrosurgical excision procedure. Obstet Gynecol 2002;99:188-92.

15. Janicek MF, Averette HE. Cervical cancer. prevention, diagnosis, and therapeutics. CA Cancer J Clin 2001;51:92-114.

16. Jemal A, Murray T, Samuels A, Ghafoor A, Ward E, Thun MJ. Cancer statistics, 2003. CA Cancer J Clin. 2003;53:5-26. 